\title{
A new radiographic classification of fifth distal metacarpal fractures
}

\author{
Ertuğrul Şahin ${ }^{1}$ (D)
}

Received: 22 April 2021 / Accepted: 9 June 2021 / Published online: 30 June 2021

(c) Royal Academy of Medicine in Ireland 2021, corrected publication 2022

\begin{abstract}
Background The fifth metacarpal fractures are the most common in all of hand fractures. To our knowledge, the classification of the fifth distal metacarpal bone fractures has not been studied.

Aims The aim of this study was to describe a new classification system based on x-ray and to evaluate its reliability and reproducibility.

Material and methods A total of 166 fifth distal metacarpal fractures were identified for classification and recorded. Two orthopedic surgeons reviewed and categorized them according to a newly designed classification. twice 1 month apart. Reliabilities of intra- and inter-observer were calculated with Spearman's rho correlation coefficient.

Results Mean values of inter and intra-observer reliability were excellent $(p=0.85)$ and substantial $(p=0.70)$, respectively. In 166 patients (163 males and 3 females), concerning the percentage of the distribution of fracture types, the most common type was Type I accounted for 81 (48.8\%) followed by Type II 70 (42.2\%), Type III 11 (6.6\%), and Type IV 4 (2.4\%). Type Ia was the most prevalent among all groups.

Conclusion This study represented a unique classification system for fractures of the distal part of the fifth metacarpal bone. Categorization in radiographs might provide ideas regarding the prognosis and clinical outcomes of fracture patterns. Therefore, this study could guide future investigations to determine the first-line treatment of fifth distal metacarpal fracture patterns using this classification and help form a common language among surgeons concerning their treatment options.
\end{abstract}

Keywords Boxer $\cdot$ Classification $\cdot$ Fifth metacarpal $\cdot$ Fracture $\cdot$ Hand

\section{Introduction}

Fractures of the metacarpals and phalanges are the most common fractures of the upper extremities [1,2]. The classification of the fifth metacarpal bone has been based on anatomical regions (head, neck, shaft, and base) [3]. Fractures of the metacarpal head are rare and mostly seen in the index finger, and they are expected to become intra-articular. Some types of metacarpal head fractures include epiphyseal, avulsion, comminuted, and boxer fractures with articular extension [4]. In addition to head fractures, neck fractures, especially in the fourth and fifth fingers (boxer's fractures), are the most common types of metacarpal fractures, accounting for $20 \%$ of all hand fractures $[1,5,6]$. Metacarpal shaft fractures are classified into three types: transverse, oblique/

Ertuğrul Şahin

ertugrulsahinn@hotmail.com

1 Kemalpaşa State Hospital, Sekiz Eylul Mahallesi, Kırovası küme boulevard, 8/1 street, 35730 Kemalpaşa, Izmir, Turkey spiral, and comminuted [4]. The AO Foundation and Orthopaedic Trauma Association (AO/OTA) classification of metacarpal fractures includes three parts: head, shaft, and base. Head fractures are classified into subcapital and intraarticular fractures [7].

Classification systems are important to create a common language of evaluation and discussions, so they are necessary, especially in clinical research. They provide comparisons and provide ideas about prognosis. The most suitable classification systems must be reliable and reproducible [8]. The AO classification and anatomic classification have been widely used to identify fracture types and make decisions regarding the treatment of fractures, but some fracture types cannot be placed into one of the subgroups of these classification systems. For these reasons, we developed a classification system of fifth distal metacarpal fractures based on X-ray findings. The hypothesis of the study was that this definition would be simpler and more descriptive, would have high reliability rates and would lead to the development of new treatment guidelines. 


\section{Materials and methods}

\section{Patients}

Radiographic images of fifth distal metacarpal fractures were obtained at our institution from June 2015 to June 2020. Patients were enrolled through a manual search of records from consultations and outpatient clinics. Patients under 18 years of age, with a history of previous fifth distal metacarpal fractures or with fractures involving other bones of the hand (except the fourth metacarpal), were excluded. A total of 166 fifth distal metacarpal fractures were identified for classification and recorded. Two orthopedic surgeons reviewed and categorized them according to a newly designed classification. Each radiograph was assessed twice 1 month apart. The assessment of anteroposterior and oblique views was performed on the Sectra Uniview digital imaging platform (version 21.2.11.6289, Linköping, Sweden). This study was approved by a non-interventional clinical research ethics board with protocol number 2020/13-08 on June 15, 2020.

\section{Radiographic examination: classification method}

Standard anteroposterior and oblique radiographs were obtained. The distal part of the fifth metacarpal bone was divided into four subgroups with three lines on anteroposterior radiographs: Line 1: between tuberosities at the most prominent parts of the head; Line 2: at the transition zone starting to appear in both the lateral and medial cortices; and Line 3: at the isthmus of the diaphysis (Fig. 1). The classification of the fracture type was made on anteroposterior radiography by determining its location according to these lines. Subtypes were also categorized according to the fracture line in the oblique view. Type I was located at the distal side of line one (between two tubercles), Type II was located between lines one and two (at the junction of the head and shaft of the metacarpal bone), and Types III

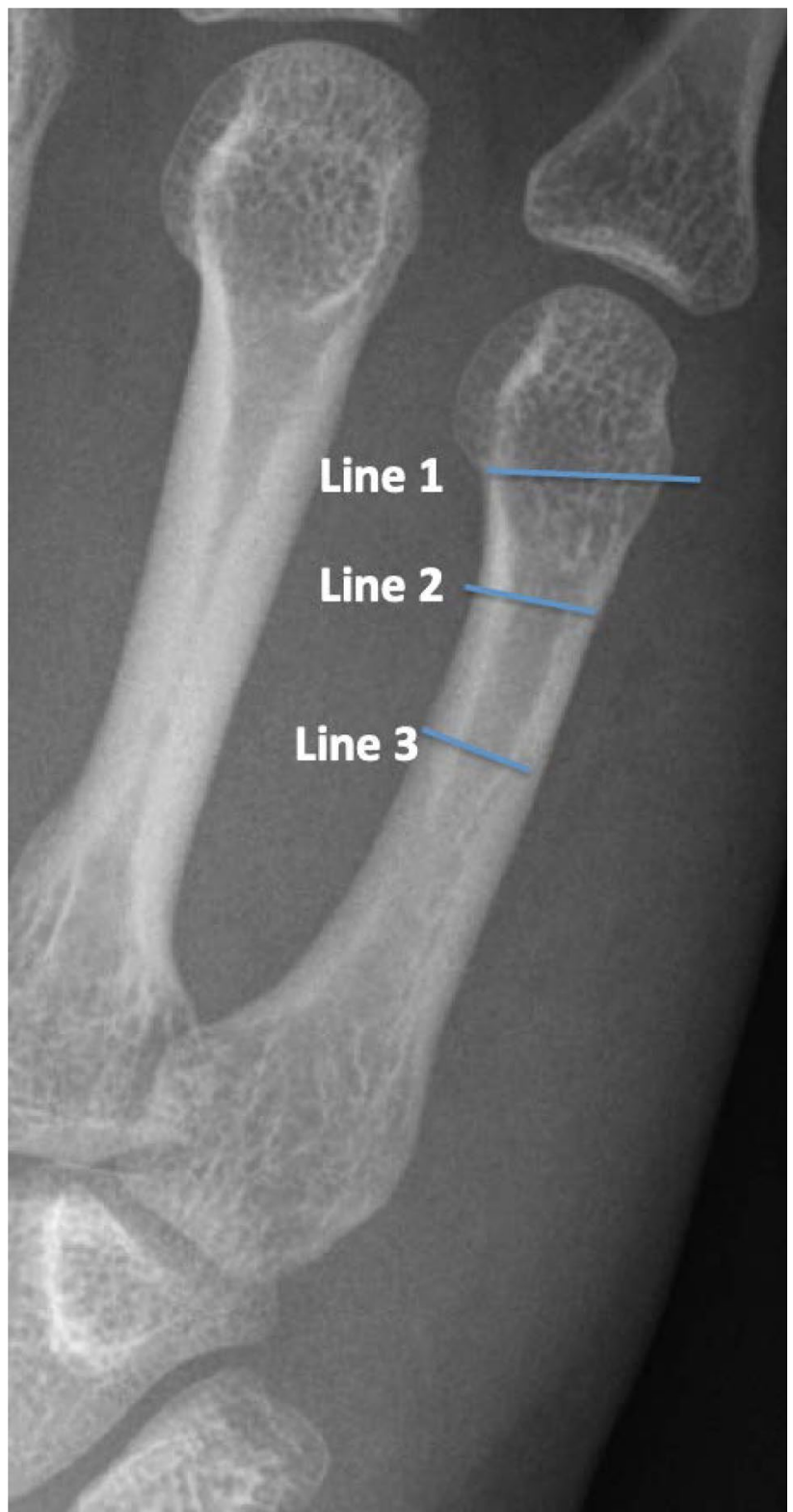

Fig. 1 Anteroposterior view showing 3 parts of fifth distal metacarpal bone

Table 1 New radiographic classification of the fifth distal metacarpal fractures $(N=166)$

\begin{tabular}{ll}
\hline Types & Radiographic description \\
\hline Type I & Distal part of line one \\
Type Ia (Boxer's fracture) & Transverse fracture between tubercles \\
Type Ib & Fracture starting at the articular surface of head, extend vertically to proximal \\
Type Ic & Comminuted fracture \\
Type II & Bicortical fracture between line one and line two \\
Type IIa & Starting from lateral tubercle, extending proximally through the medial cortex, lateral cortex intact \\
Type IIb & Starting from lateral tubercle, extending proximally through the medial cortex, lateral cortex broken \\
Type IIc & Transverse fracture through line two \\
Type III & Bicortical fracture at the isthmus of bone \\
Type IV & Type III + fourth metacarpal shaft fracture \\
\hline
\end{tabular}



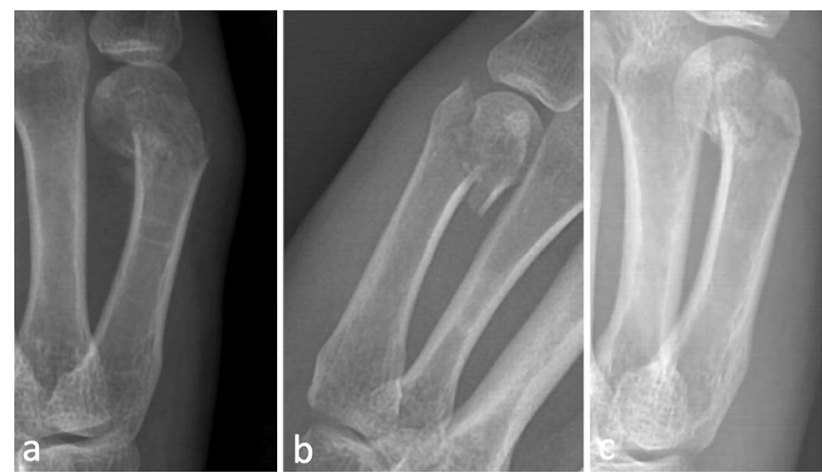

Fig. 2 Oblique view showing a Type Ia, transverse, between tubercles; b Type Ib, articular surface of head to proximal; and c Type Ic, comminuted, fracture of the fifth distal metacarpal bone

and IV were located at the isthmus of the metacarpal bone. All types of fractures and their radiographic descriptions are shown in Table 1.

Type I fractures consist of three subtypes, as shown in Fig. 2. Type Ia, also called boxer's fracture, consists of transverse fractures between the tubercles. Type Ib consists of fractures starting at the articular surface of the head and extending vertically to the proximal surface. Type Ic is a comminuted fracture of the metacarpal head.

Type II fractures consist of three subtypes, as shown in Fig. 3. Type IIa starts from the lateral tubercle and extends proximally through the medial cortex, but the lateral cortex is intact. Type IIb is the same as type IIa, but the lateral cortex is also broken. Type IIc consists of transverse fractures through line two.

Type III consists of bicortical fractures at the isthmus of the bone (Fig. 4). Type IV consists of Type III plus fourth metacarpal shaft fracture (Fig. 5). Using these criteria, each radiograph was classified separately by two investi-
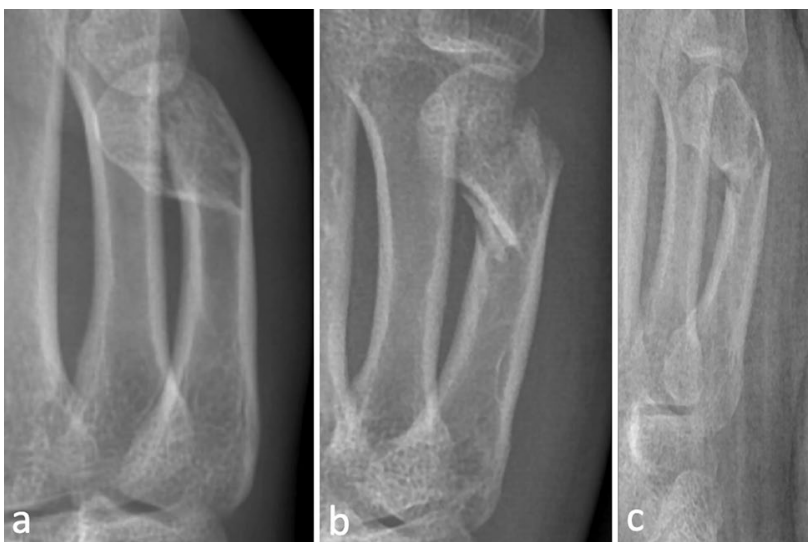

Fig. 3 Oblique view showing a Type IIa, lateral cortex intact; b Type IIb, lateral cortex broken; and c Type IIc, transverse, fracture of the fifth distal metacarpal bone

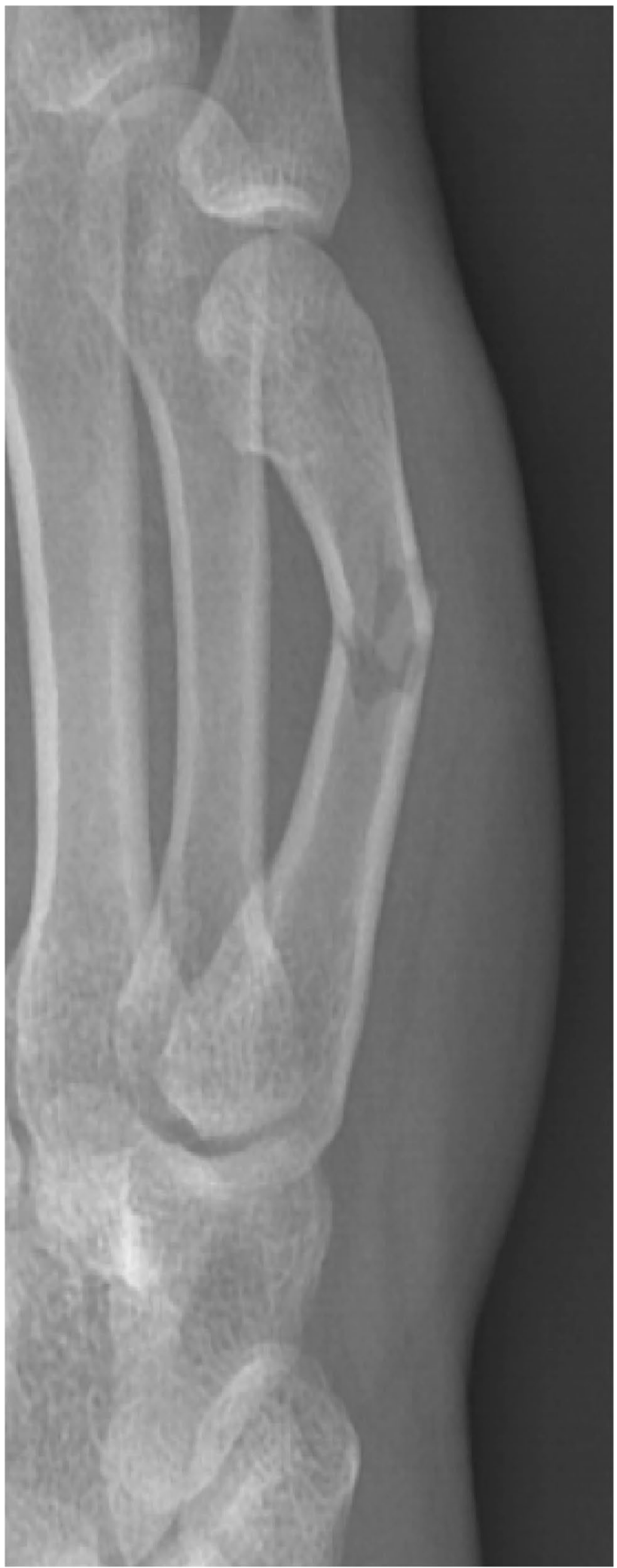

Fig. 4 Oblique view showing a Type III, bicortical fracture at the isthmus, fracture of the fifth distal metacarpal bone 


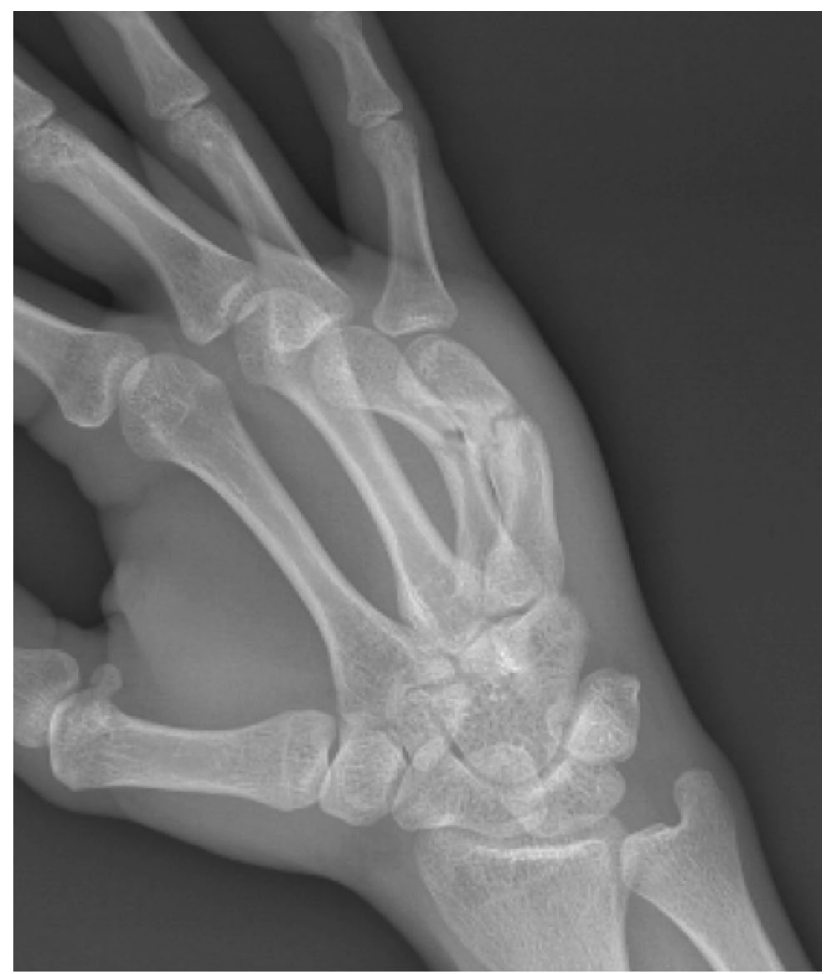

Fig. 5 Oblique view showing a Type IV, Type III + fourth metacarpal shaft fracture, fracture of the fifth distal metacarpal bone

gators, and the prevalence of each subtype was recorded and analyzed. The final results were determined by jointly reviewing radiographs that were classified differently in an open discussion.

The intraobserver and interobserver correlations were calculated with the intraclass correlation coefficient (ICC) and p values [9]. Spearman's rho (r) correlation coefficient was used to analyze correlations between intraobserver agreements. Spearman's rho (p) values less than 0.2 are slight, $0.21-0.40$ are fair, $0.41-0.60$ are moderate, $0.61-0.80$ are substantial, and more than 0.80 are

Table 2 Prevalence of radiographic classification of the fifth distal metacarpal fracture

\begin{tabular}{cl}
\hline Types & $\begin{array}{l}\text { Total number of } \\
\text { cases }(\%)\end{array}$ \\
\hline I & $81(48.8)$ \\
Ia & $71(42.8)$ \\
Ib & $6(3.6)$ \\
Ic & $4(2.4)$ \\
II & $70(42.2)$ \\
IIa & $34(20.5)$ \\
IIb & $27(16.3)$ \\
IIc & $9(5.4)$ \\
III & $11(6.6)$ \\
IV & $4(2.4)$
\end{tabular}

Table 3 Intra and inter-observer reliability values

\begin{tabular}{llllll}
\hline Groups & \multicolumn{2}{l}{ First round } & & \multicolumn{2}{l}{ Second round } \\
\cline { 2 - 3 } & ICC & Meaning & & ICC & Meaning \\
\hline Inter-observer & 0.81 & Excellent & & 0.89 & Excellent \\
Intra-observer & & & & \\
Surgeon 1 & 0.73 & Substantial & & \\
Surgeon 2 & 0.67 & Substantial & & \\
\hline
\end{tabular}

almost perfect agreements [10]. An ICC value $<0.40$ indicates poor agreement, $0.40-0.59$ indicates fair agreement, $0.60-0.75$ indicates good agreement, and above 0.75 indicates excellent agreement [11]. All analyses were performed using IBM SPSS Statistics 22.0 (SPSS Inc., Chicago, IL). A value below 0.05 was accepted as statistically significant.

\section{Results}

A total of 166 patients (163 males and 3 females) were included. The mean age of all patients was $30.5 \pm 12$ (18-64) years. Of all the cases, $15.1 \%$ included the left side. The percentage of the distribution of fracture types is shown in Table 2. Almost half of all fractures were Type I, followed by Type II (42.2\%). Type Ia was the most common type in all groups and subgroups, accounting for 71 cases (42.8\%), followed by Type IIa, accounting for 34 cases (20.5\%). The intraobserver and interobserver correlations were positive among surgeons in both the first and second rounds (Table 3). The interobserver correlation in the second round was higher than that in the first round. However, the intraobserver correlations for both rounds were close to each other. The mean values of interobserver and intraobserver reliability were excellent $(p=0.85)$ and substantial $(p=0.70)$, respectively.

\section{Discussion}

In this study, the interrater correlation coefficients were substantial, and the intrarater correlation was excellent. These results showed that the proposed classification of fifth distal metacarpal fractures was reliable.

The usefulness of classification systems is mostly related to having good interobserver and intraobserver reliability, being reproducible, helping select the appropriate treatment, being simple, being easy to use in clinical practice and providing information about prognosis [12-14]. Orthopedic surgeons, especially those dealing 
with trauma, use classification systems to determine the treatment modality and prognosis. Furthermore, newly designed classifications should have high reliability rates to be widespread. The AO/OTA subdivided bones with articulations at both ends and a segment of cortical bone into three parts: proximal, shaft, and distal [15]. However, no studies have focused on the distal part of the fifth metacarpal bone. In addition, Szwebel et al. [16] observed that the OTA classification has poor reliability. Based on these findings, our study had advantages in classifying fifth metacarpal distal fractures, which has never been done before, and has high reliability rates.

Despite the high prevalence of metacarpal fractures, there is still no consensus regarding the most appropriate management $[17,18]$. For conservative management, studies report several different treatment options ranging from buddy taping to cast with different periods for immobilization [19]. Surgical procedures are usually preferred for patients with comminuted, open, irreducible, or intraarticular fractures, polytrauma, rotational deformity, and/or shortening of bone to obtain optimal results $[20,21]$. The selection of a treatment option is still controversial because there have been no definitions of distal metacarpal fractures, which leads to some bias in clinical studies and, of course, the results.

Interrater and intrarater reliability tests are preferred for radiographic classification to determine the reproducibility by assessing the same views several times. The increased correlation between researchers in the second round of evaluation made us believe that the researchers adapted to the criteria of the scoring system and learned to interpret them better, although the images were presented in a shuffled order with no definitive signs on them. There is always a possibility of inconsistency between observers during radiographic evaluations. In this study, high intraobserver correlation values showed that this system could overcome this issue.

In this study, the classification system presented 4 types of fracture patterns: Type Ia, Ib, and Ic; Type IIa, IIb, and IIc; and Type III and Type IV. These patterns were distinguished according to the location of the bone and the direction of the fracture line.

This study was limited to only radiographic examination and did not address the prognosis of fracture and treatment options. Therefore, additional research is needed to correlate the fracture type with treatment modalities and prognosis. Like most retrospective studies, the results might be influenced by methodologic factors that could affect the quality of the study.

\section{Conclusion}

In conclusion, we presented a unique classification system for fractures of the distal part of the fifth metacarpal bone. Categorization in radiographs might provide ideas regarding the prognosis and clinical outcomes of fracture patterns. Therefore, this study could guide future investigations to determine the first-line treatment of fifth distal metacarpal fracture patterns using this classification and help form a common language among surgeons concerning their treatment options.

Acknowledgements I want to thank Mustafa Vezirhuyuk, MD, who evaluate the radiographics and classified them.

Author contribution ES: Design of the work; collection, analysis, and interpretation of data for the work; drafting the work, final approval of the version to be published.

Data availability The data that support the findings of this study are available on request from corresponding author (Ertugrul Sahin). The data are not publicly available due to restrictions.

\section{Declarations}

Ethics approval Dokuz Eylul University Non-interventional Clinical Research Ethics Board with protocol number 2020/13-08 on June 15, 2020.

Informed consent The research is based on data which is open to public. Patient informed consent was not required.

Competing interests The authors declare no competing interests.

\section{References}

1. Chung KC, Spilson SV (1993) The frequency and epidemiology of hand and forearm fractures in the United States. Hand Surg 26:908-915

2. Hove LM (1993) Fractures of the hand. Distribution and relative incidence. Scand J Plast Reconstr Surg Hand Surg 27:317-319

3. Gudmundsen TE, Borgen L (2009) Fractures of fifth metacarpal. Acta Radiol 50(3):296-300

4. Charles SD (2017) Fractures of the metacarpals and phalanges. In: Hand Wolfe SW, Hotchkiss RN, Pederson WC, Kozşn SH, Cohen MS (ed), Elsevier, New York

5. Kural C, Alkas L, Tuzun S et al (2011) Anger scale and anger types of patients with fifth metacarpal neck fractures. Acta Orthop Traumatol Turc 45(5):312-315

6. Greer MA (2008) Incidence of metacarpal fractures in U.S. soldiers stationed in South Korea. J Hand Ther 21(2):137-141

7. Jupiter JB, Ring DC (2005) AO Manual of Fracture ManagementHand and Wrist. Georg Thieme Verlag, New York 
8. Leonetti D, Tigani D (2017) Pilon fractures: a new classification system based on CT-scan. Injury 48(10):2311-2317

9. Fleiss JL, Cohen J (1973) The equivalence of weighted kappa and intraclass correlation coefficient as measures of reliability. Educ Psychol Meas 33:613-619

10. Altman DB (1999) Practical statistics for medical research. Chapman \& Hall, London

11. Landis JR, Koch GG (1977) The measurement of observer agreement for categorical data. Biometrics 33:159-174

12. Karanicolas PJ, Bhandari M, Kreder H et al (2009) Collaboration for outcome assessment in surgical trials (COAST) musculoskeletal group (2009) evaluating agreement: conducting a reliability study. J Bone Joint Surg Am 91(Suppl 3):99-106

13. Ramappa M, Bajwa A, Singh A et al (2010) Interobserver and intraobserver variations in tibial pilon fracture classification systems. Foot (Edinb) 20(2-3):61-63

14. Mehlhorn AT, Zwingmann J, Hirschmüller A et al (2014) Radiographic classification for fractures of the fifth metatarsal base. Skeletal Radiol 43:467-474

15. Meinberg EG, Agel J, Roberts CS et al (2018) Fracture and dislocation classification compendium-2018. J Orthop Trauma 32(1):S1-S170

16. Szwebel JD, Ehlinger V, Pinsolle V et al (2010) Reliability of a classification of fractures of the hand based on the $\mathrm{AO}$ comprehensive classification system. J Hand Surg Eur Vol 35(5):392-395

17. Kollitz KM, Hammert WC, Vedder NB et al (2014) Metacarpal fractures: treatment and complications. Hand (NY) 9(1):16-23

18. Diaz-Garcia R, Waljee JF (2013) Current management of metacarpalfractures. Hand Clin 29(4):507-518

19. Retrouvey H, Morzycki A, Wang AMQ et al (2018) Are we over treating hand fractures? current practice of single metacarpal fractures. Plast Surg (Oakv) 26(3):148-153

20. Wong VW, Higgins JP (2017) Evidence-based medicine: management of metacarpal fractures. Plast Reconstr Surg 140(1):140e-151e

21. Sahu A, Gujral SS, Batra S et al (2012) The current practice of the management of little finger metacarpal fractures-a review of the literature and results of a survey conducted among upper limb surgeons in the United Kingdom. Hand Surg 17(1):55-63

Springer Nature or its licensor holds exclusive rights to this article under a publishing agreement with the author(s) or other rightsholder(s); author self-archiving of the accepted manuscript version of this article is solely governed by the terms of such publishing agreement and applicable law. 